\title{
Smart Magnetically Responsive Hydrogel Nanoparticles Prepared by a Novel Aerosol-Assisted Method for Biomedical and Drug Delivery Applications
}

\author{
Ibrahim M. El-Sherbiny, ${ }^{1,2}$ and Hugh D. C. Smyth ${ }^{2}$ \\ ${ }^{1}$ Polymer Laboratory, Chemistry Department, Faculty of Science, Mansoura University, 35516 Mansoura, Egypt \\ ${ }^{2}$ Division of Pharmaceutics, College of Pharmacy, The University of Texas at Austin, Austin, TX 78712, USA \\ Correspondence should be addressed to Ibrahim M. El-Sherbiny, imelsherbiny@gmail.com
}

Received 27 August 2010; Accepted 14 December 2010

Academic Editor: Xingmao Jiang

Copyright (C) 2011 I. M. El-Sherbiny and H. D. C. Smyth. This is an open access article distributed under the Creative Commons Attribution License, which permits unrestricted use, distribution, and reproduction in any medium, provided the original work is properly cited.

\begin{abstract}
We have developed a novel spray gelation-based method to synthesize a new series of magnetically responsive hydrogel nanoparticles for biomedical and drug delivery applications. The method is based on the production of hydrogel nanoparticles from sprayed polymeric microdroplets obtained by an air-jet nebulization process that is immediately followed by gelation in a crosslinking fluid. Oligoguluronate (G-blocks) was prepared through the partial acid hydrolysis of sodium alginate. PEG-grafted chitosan was also synthesized and characterized (FTIR, EA, and DSC). Then, magnetically responsive hydrogel nanoparticles based on alginate and alginate/G-blocks were synthesized via aerosolization followed by either ionotropic gelation or both ionotropic and polyelectrolyte complexation using $\mathrm{CaCl}_{2}$ or PEG-g-chitosan/ $\mathrm{CaCl}_{2}$ as crosslinking agents, respectively. Particle size and dynamic swelling were determined using dynamic light scattering (DLS) and microscopy. Surface morphology of the nanoparticles was examined using SEM. The distribution of magnetic cores within the hydrogels nanoparticles was also examined using TEM. In addition, the iron and calcium contents of the particles were estimated using EDS. Spherical magnetic hydrogel nanoparticles with average particle size of $811 \pm 162$ to $941 \pm 2 \mathrm{~nm}$ were obtained. This study showed that the developed method is promising for the manufacture of hydrogel nanoparticles, and it represents a relatively simple and potential low-cost system.
\end{abstract}

\section{Introduction}

Over the last two decades, stimuli-responsive "smart" hydrogels, which can respond reversibly to external stimuli, such as $\mathrm{pH}$, temperature, and electric field, have attracted a great deal of interest due to their potential applications in various fields especially in controlled drug delivery [1]. In the recent years, a significant body of research has focused on the development of biocompatible magnetically responsive nanoparticles for various drug delivery and biomedical applications such as magnetic drug targeting, enzyme immobilization, hyperthermia anticancer treatment, and the magnetic resonance imaging for clinical diagnosis [2-9]. The efficiency of magnetic nanoparticles in most of these applications depends particularly on the particle size distribution and the morphology of the polymer/magnetic nanoparticles $[10,11]$.
The magnetically responsive hydrogel nanoparticles with high-saturation magnetization and high susceptibility showed a good ability to trigger drug release upon applying external magnetic stimuli [12]. The major advantage of this drug delivery technology is attributed to the magnetic characteristics of the carrier system, which can be controlled remotely, and the biocompatibility of both the encapsulated iron oxide nanoparticles (e.g., magnetite $\left(\mathrm{Fe}_{3} \mathrm{O}_{4}\right)$ and maghemite $\left.\left(\gamma-\mathrm{Fe}_{2} \mathrm{O}_{3}\right)\right)$ and the polymeric hydrogel matrices. Superparamagnetic iron oxide nanoparticles (SPIONs) which can be easily magnetized and concentrated in a specific site by applying an external magnetic field and redispersed again once the magnetic field is removed have also found a recent considerable interest for drug delivery purposes [13].

The production of hydrogel nanoparticles such as acrylate-based hydrogels typically utilizes bioincompatible solvents such as dimethylformamide and acetone. Moreover, 
the processing can be costly, and particle size distributions are typically very broad. Thus there is a need for new processes that can efficiently produce nanoparticles with desirable particle size in addition to exploring new biocompatible polymeric systems that ensure the production of the nanoparticles with optimal characteristics using biocompatible solvents relevant to actual clinical use. This current contribution involves the development of a novel spray gelation-based method (aerosol-mediated method) which has been utilized to synthesize a series of magnetically responsive "smart" alginate-based hydrogel nanoparticles for biomedical and drug delivery applications. This method is based on the production of hydrogel nanoparticles from sprayed polymeric microdroplets obtained through an air-jet nebulization process that is immediately followed by a gelation step in a crosslinking fluid. The synthesized magnetically responsive hydrogel nanoparticles are comprised of either sodium alginate or a combination of sodium alginate with oligoguluronate (G-blocks) aerosolized as microdroplets followed by either ionotropic gelation in aqueous $\mathrm{Ca}^{2+}$ solution or both ionotropic and polyelectrolyte complexation in aqueous solution of PEG-g-chitosan/ $\mathrm{CaCl}_{2}$ as crosslinkers.

Sodium alginate is a natural nontoxic biodegradable polyanionic copolymer consist of 1,4-linked $\beta$-Dmannuronic acid (M) and $\alpha$-L-guluronic acid (G) residues arranged either as consecutive blocks or in a random distribution. Sodium alginate has a unique ability to form hydrogels in the presence of divalent cations such as $\mathrm{Ca}^{2+}$, $\mathrm{Ba}^{2+}$, or $\mathrm{Sr}^{2+}$ ions [14] through lateral association of chain segments (ionotropic crosslinking). It has been reported that this ionotropic chelation/crosslinking occurs particularly between the cations and the G-blocks of alginate as described by the "egg-box" model $[15,16]$ in which each divalent cation interacts with two adjacent $G$ residues as well as with two $G$ residues in an opposing alginate chain. Therefore, the gel strength of the alginate-based hydrogels depends mainly on the $\mathrm{G}$ content in addition to other parameters such as the divalent cation concentration and the concentration and molecular weight of the alginate. It has been also found that sodium alginate has a strong ability to form hydrogels via polyelectrolyte complexation with cationic polymers such as chitosan (Cs) and its derivatives [14].

Chitosan (Cs), a cationic polymer obtained through the alkaline $\mathrm{N}$-deacetylation of chitin, has various desirable properties such as nontoxicity, biodegradability, and biocompatibility [17]. Grafting of various synthetic polymers onto the Cs backbone can further improve its characteristics and accordingly expand its potential applications. One of these polymers that is commonly grafted onto Cs is the poly(ethylene glycol) (PEG). PEG is a water-soluble nontoxic and biocompatible polymer [18]. Owing to the characteristics of PEG, the development of polymeric micro- and nanohydrogel particles based on PEG graft copolymerized onto Cs (PEG-g-Cs) has received increased attention in many applications specifically for drug delivery purposes [19-22].

In this study, oligoguluronate residues (G-blocks) were prepared through partial acid hydrolysis of sodium alginate. PEG-g-Cs was also synthesized and characterized. Then a new series of magnetic hydrogel nanoparticles was developed using a new method. The magnetic hydrogel nanoparticles are based on a combination of alginate with the G-blocks and crosslinked either with $\mathrm{Ca}^{2+}$ (ionotropic gelation) or through polyelectrolyte complexation with the cationic PEG-g-Cs.

\section{Materials and Methods}

2.1. Materials. Chitosan (medium MW, \%N-deacetylation; $76.4 \%$, as determined by FTIR spectroscopy and elemental analysis), monomethoxy-poly-(ethylene glycol) (m-PEG, Mn $5 \mathrm{kDa}$ ), succinic anhydride, and 1-hydroxybenzotrizole (HOBt) were supplied by Aldrich (USA). 1-Ethyl-3(3-dimethylaminopropyl) carbodiimide hydrochloride (EDC.HCL) was obtained from Fluka Chemical Corp. (Milwaukee, WI, USA). 4-Dimethylaminopyridine (DMAP) was provided by Sigma (St. Louis, MO, USA). Sodium alginate (low viscosity; $250 \mathrm{cps}$ for a $2 \%$ solution at $25^{\circ} \mathrm{C}$ ), phthalic anhydride, dioxane, triethyl amine, and dimethyl formamide (DMF) were obtained from Sigma-Aldrich (St Louis, MO, USA). The superparamagnetic iron oxide nanoparticles (SPIONs) and fluidMAG-D of size $100 \mathrm{~nm}$ were purchased from Chemicell (Berlin, Germany). Ethanol, phosphate buffer saline (PBS pH 7.4), and all other reagents were of analytical grade and used as received.

\subsection{Methods}

2.2.1. Preparation of Oligoguluronate (G-Blocks). The oligoguluronate residues (G-blocks) were prepared using a method similar to that described by Haug et al. [23, 24]. Briefly, sodium alginate $(10 \mathrm{~g})$ was dissolved in distilled water and made up to $1 \mathrm{~L}$ with $0.3 \mathrm{M} \mathrm{HCl}$. The mixture was then heated at $100^{\circ} \mathrm{C}$ for $6 \mathrm{~h}$. The oligomannuronate and oligoguluronate homopolymeric blocks that remained intact as solid residues were collected via centrifugation for $5 \mathrm{~min}$ at $4000 \mathrm{rpm}$, washed, and resuspended in distilled water. The residue was dissolved by the dropwise addition of $0.3 \mathrm{M} \mathrm{NaOH}$. Afterwards, $\mathrm{NaCl}$ was added to make up a final concentration of $0.5 \%(\mathrm{w} / \mathrm{v})$. Ethanol (2 volumes) was added and the resulting precipitate was collected by centrifugation for $5 \mathrm{~min}$ at $4000 \mathrm{rpm}$. The precipitate was then washed and redissolved in water, and the $\mathrm{pH}$ was adjusted to 2.85 with $1 \mathrm{M} \mathrm{HCl}$. At this $\mathrm{pH}$ value, the oligoguluronate blocks were precipitated and then collected leaving the oligomannuronate blocks in solution. The oligoguluronate fractions were desalted and freeze-dried.

2.2.2. Preparation of Cs Grafted with PEG. The PEG-g-Cs copolymer was synthesized as described in our earlier study [25] and summarized as follows.

(i) Preparation of N-Phthaloyl Cs (NPHCs). Phthalic anhydride ( $44.8 \mathrm{~g}, 5 \mathrm{~mol}$ equivalent to pyranose rings) was allowed to react with $10 \mathrm{~g}$ of Cs in $200 \mathrm{ml}$ DMF at $130^{\circ} \mathrm{C}$ under dry nitrogen atmosphere for $8 \mathrm{~h}$. The resulting NPHCs (pale brown solid) was then collected by filtration after precipitation in ice-water, washed with methanol, and dried under vacuum at $40^{\circ} \mathrm{C}$. 
(ii) Preparation of m-PEG-COOH. m-PEG (100 g, $20 \mathrm{mmol})$, DMAP $(2.44 \mathrm{~g}, 20 \mathrm{mmol})$, triethylamine $(2.02 \mathrm{~g}$, $20 \mathrm{mmol})$, and succinic anhydride ( $2.4 \mathrm{~g}, 24 \mathrm{mmol})$ were dissolved in dry dioxane $(350 \mathrm{ml})$ and then stirred for 2 days at room temperature under a dry nitrogen atmosphere. The dioxane was evaporated using a rotary evaporator, and the residue was taken up in $\mathrm{CCl}_{4}$, filtered, and precipitated by diethyl ether to produce m-PEG-COOH (white powder).

(iii) Preparation of PEG-g-NPHCs. m-PEG-COOH (37.9 g) and NPHCs (5.0 g, $0.4 \mathrm{~mol}$ equivalent to m-PEG-COOH) were dissolved in $75 \mathrm{ml}$ of DMF. The HOBt $(3.4 \mathrm{~g}, 3 \mathrm{~mol}$ equivalent to m-PEG-COOH) was then added, as a catalyst, with stirring at room temperature until obtaining a clear solution. Afterwards, the EDC. $\mathrm{HCl}(4.25 \mathrm{~g}, 3$ mol equivalent to $\mathrm{m}-\mathrm{PEG}-\mathrm{COOH}$ ) was added, and the mixture was stirred overnight at room temperature. A purified PEG-g-NPHCs copolymer $(5.47 \mathrm{~g}$, white solid) was obtained after dialysis of the reaction mixture against distilled water and washing with ethanol.

(iv) Preparation of PEG-g-Cs. The PEG-g-NPHCs (4.0 g) was dissolved in $15 \mathrm{ml}$ of DMF and heated to $100^{\circ} \mathrm{C}$ with stirring under nitrogen. Afterwards, $20 \mathrm{ml}$ of hydrazine monohydrate was added, and the reaction was continued for $2 \mathrm{~h}$. The resulting PEG-g-Cs copolymer was purified via dialysis against a mixture of deionized ethanol and water $(1: 1)$ then dried under vacuum at $40^{\circ} \mathrm{C}$.

NPHCs. FTIR $\left(v_{\max }, \mathrm{cm}^{-1}\right): 3281(\mathrm{OH}$ stretching and $\mathrm{NH}$ bending), 2961 (C-H stretching), 1775, and 1698 ( $\mathrm{C}=\mathrm{O}$ anhydride), 1395 ( $\mathrm{C}=\mathrm{C}$, phthaloyl), 1058 (C-O, pyranose), and 732 (aromatic ring of phthaloyl). Elemental analysis (EA), $\left(\mathrm{C}_{8} \mathrm{H}_{13} \mathrm{NO}_{5}\right)_{0.2363}\left(\mathrm{C}_{6} \mathrm{H}_{11} \mathrm{NO}_{4}\right)_{0.016}\left(\mathrm{C}_{14} \mathrm{H}_{13} \mathrm{NO}_{6}\right)_{0.747}$, Anal. calculated $(\mathrm{DS}=0.98)(\%): \mathrm{C}, 55.74 ; \mathrm{H}, 4.84 ; \mathrm{N}$, 5.23, found (\%): C, 60.31; H, 4.83; N, 4.92. m-PEG-COOH; FTIR $\left(v_{\max }, \mathrm{cm}^{-1}\right) 3496(\mathrm{OH}$ stretching), $2882(\mathrm{C}-\mathrm{H}$ stretching), 1733 ( $\mathrm{C}=\mathrm{O}$ of carboxylic group), and 1102 (C-O-C stretching); EA, $\left(\mathrm{C}_{231} \mathrm{H}_{460} \mathrm{O}_{117}\right)$, Anal. calculated (\%): $\mathrm{C}, 54.35$, and $\mathrm{H}, 9.02$, found (\%): $\mathrm{C}, 56.8$, and $\mathrm{H}$, 9.19. PEG-g-NPHCs $(5.47 \mathrm{~g})$; FTIR $\left(v_{\max }, \mathrm{cm}^{-1}\right) 3423(\mathrm{OH}$ stretching and $\mathrm{NH}$ bending), 2879 (C-H stretching), 1736 ( $\mathrm{C}=\mathrm{O}$ ester and anhydride), $1703(\mathrm{C}=\mathrm{O}$ anhydride), 1096 (C-O-C stretching), and 723 (aromatic ring of phthaloyl). EA, found (\%): C, 56.16; H, 4.69; N, 5.15. PEG-g-Cs; FTIR $\left(v_{\max }, \mathrm{cm}^{-1}\right) 3312(\mathrm{OH}$ stretching, $\mathrm{NH}$ bending, and intermolecular H-bonding), 2879 (C-H stretching), 1708 $(\mathrm{C}=\mathrm{O}$ ester), and 1096 (C-O-C stretching). EA, found (\%): C, $40.46 ; \mathrm{H}, 4.71 ; \mathrm{N}, 14.44$.

2.2.3. Characterization of the Synthesized Polymers. The elemental analysis (EA) was carried out using Costech ECS4010 elemental analyzer coupled to a Thermo-Finnigan Delta Plus isotope ratio mass spectrometer. The FTIR spectra were recorded using Nicolet 6700 FTIR spectrometer, and the differential scanning calorimetry (DSC) was carried out using a DSC 2920 (Modulated DSC, TA Instruments) in a nitrogen atmosphere with a temperature range of $-40^{\circ} \mathrm{C}$ to $400^{\circ} \mathrm{C}$ at a heating rate of $10^{\circ} \mathrm{C} \min ^{-1}$ for preweighed samples of $10-15 \mathrm{mg}$. The peaks' temperatures and the enthalpy values were recorded.

\subsubsection{Preparation of the Magnetic Hydrogel Nanoparticles} Using a Novel Aerosol-Mediated Method. The magnetic hydrogel nanoparticles were prepared using a novel aerosolassisted method. This method depends on the manufacture of hydrogel nanoparticles from sprayed polymeric microdroplets obtained by an air-jet nebulization process which is immediately followed by gelation/hardening in a collecting crosslinking fluid. Specifically, $2 \% \mathrm{w} / \mathrm{v}$ aqueous solutions of either sodium alginate or mixtures of sodium alginate with oligoguluronate of different compositions (Table 1 ) were prepared. Also, a $0.1 \% \mathrm{w} / \mathrm{v}$ aqueous solution of the synthesized copolymer, PEG-g-Cs, was prepared by dissolving it in $10 \mathrm{ml}$ of $0.06 \mathrm{M}$ acetic acid and made up to the precalculated volume with distilled water. Predetermined volumes of the SPIONs suspensions were added to the alginate or alginate/oligoguluronate solutions (3\% SPIONs based on the total polymer weight) and homogenously distributed by sonication for $2 \mathrm{~min}$. Then, the polymer/SPIONs mixtures were transferred to an Aerotech II air-jet nebulizer and aerosolized with the aid of compressed air delivered at a controlled pressure of 30 psi. The generated aerosols of SPIONs/polymer microdroplets were directed, under vacuum, into $10 \mathrm{ml}$ of a crosslinking solution of either $\mathrm{CaCl}_{2}(0.2 \mathrm{M})$ or a $(1: 1)$ mixture of PEG-g-Cs/ $\mathrm{CaCl}_{2}$ under continuous mild stirring. The resulting suspensions of the swollen magnetic hydrogel nanoparticles were collected and freeze dried. The dried hydrogel powders were then washed with distilled water to remove any residual crosslinker and then refreezing-dried. The yield (\%) of the resulting dry powders of hydrogel nanoparticles was determined, and the powders were stored in a desiccator at room temperature until further use.

2.2.5. Determination of Hydrogel Particle Size. The average size of the prepared magnetic hydrogel nanoparticles suspended in $2 \mathrm{ml}$ of ethanol $(0.2 \% \mathrm{w} / \mathrm{v})$ was determined by dynamic light scattering (DLS) (Malvern nanosizer, Malvern Instruments Ltd., Worcestershire, UK). The size of the particles was also estimated using microscopy techniques including light microscopy (Leica DMI6000B scope) with Leica Application Suite Advanced Fluorescence 2.2.0 build 4765 software. The average diameters $(d, \mathrm{~nm})$ of about 100 magnetic hydrogel nanoparticles $(n=100)$ were recorded.

2.2.6. Surface Morphology of Hydrogel Nanoparticles. The morphology of the developed magnetic hydrogel nanoparticles was investigated by SEM (Zeiss supra 40 VP scanning electron microscope). Dry magnetic nanoparticles were mounted on aluminum stubs with double-sided conducting carbon tapes and coated with a 50/50 mixture of $\mathrm{Pt} / \mathrm{Pd}$ to minimize surface charging. The samples were scanned at an accelerating voltage of $10 \mathrm{kV}$. 
TABLE 1: Different compositions and particle size of the developed magnetic hydrogel nanoparticles.

\begin{tabular}{|c|c|c|c|c|c|c|c|}
\hline \multirow{2}{*}{ Formulation code } & \multicolumn{2}{|c|}{ Polymer aq. solution $(2 \% \mathrm{w} / \mathrm{v})$} & \multicolumn{2}{|c|}{ Crosslinking fluid } & \multicolumn{3}{|c|}{ Particle size, $(d, \mathrm{~nm}) \pm \mathrm{SD}$} \\
\hline & Alginate (w\%) & G-block (w\%) & $\mathrm{CaCl}_{2}(0.2 \mathrm{M})$ & $\begin{array}{c}\mathrm{CaCl}_{2} / \text { PEG-g-Cs } \\
(1: 1)\end{array}$ & ${ }^{1}$ Dry (DLS) & ${ }^{2}$ Dry (LM) & ${ }^{2}$ Swelled (LM) \\
\hline IA & 100 & 00 & $t$ & - & $936 \pm 40$ & $852 \pm 145$ & $1546 \pm 84$ \\
\hline IB & 100 & 00 & - & $\dagger$ & $926 \pm 13$ & $811 \pm 105$ & $1498 \pm 102$ \\
\hline IIA & 80 & 20 & t & - & $811 \pm 162$ & $770 \pm 122$ & $1571 \pm 61$ \\
\hline IIB & 80 & 20 & - & $\dagger$ & $929 \pm 12$ & $794 \pm 177$ & $1427 \pm 179$ \\
\hline IIIA & 70 & 30 & t & - & $941 \pm 2$ & $855 \pm 89$ & $1640 \pm 75$ \\
\hline IIIB & 70 & 30 & - & + & $886 \pm 2$ & $800 \pm 152$ & $1592 \pm 126$ \\
\hline
\end{tabular}

${ }^{1}$ Determined using DLS. ${ }^{2}$ Estimated using light microscopy (LM).

2.2.7. Transmission Electron Microscopy Analysis of Hydrogel Nanoparticles. A Jeol 2010F field emission transmission electron microscope (TEM) was used to obtain transmission electron micrographs of the developed magnetic hydrogel nanoparticles at accelerating voltage of $200 \mathrm{kV}$. Also, the iron and calcium contents of the particles were estimated using energy-dispersive X-ray spectroscopy (EDS) (Oxford INCA) attached to the TEM with the aid of silicon detector at an accelerating voltage of $20 \mathrm{kV}$ and a magnification of $120,000 X$.

2.2.8. Dynamic Swelling Study of Hydrogel Nanoparticles. The swelling characteristics of the developed magnetic hydrogel nanoparticles $(2-3 \mathrm{mg}$ ) in $500 \mu \mathrm{L}$ of PBS ( $\mathrm{pH} 7.4$ ) were investigated by determining the increase in the particle diameter $(d, \mathrm{~nm})$ at different time intervals; 2,5 , and $10 \mathrm{~min}$ using light microscopy (Leica DMI6000B scope) with Leica Application Suite Advanced Fluorescence 2.2.0 build 4765 software.

2.2.9. Statistical Analysis and Optimization of the Magnetic Hydrogel Nanoparticles by a $2^{k}$ Factorial Design. The obtained results were analyzed and expressed as mean \pm SD. A $2^{k}$ factorial design was used to estimate the effect of the different formulation's parameters such as the alginate $\%$ and the type of crosslinking agent on the characteristics of the developed magnetic hydrogel nanoparticles such as particle size and equilibrium swelling for further optimizations. Also, the statistical significance was analyzed using ANOVA (Statgraphics Plus version 5.0 software). Differences were considered significant at the level of $P<.05$.

\section{Results and Discussion}

3.1. Preparation of Oligoguluronate (G-Blocks). The oligoguluronate residues (G-blocks) were obtained through the partial acid hydrolysis of sodium alginate followed by fractionation of the products. The hydrolysis was achieved using dilute $\mathrm{HCl}$ in boiling water through a method similar to that described by Haug et al. [23, 24]. The partial hydrolysis process of the alginate led to two fractions; the soluble fraction consists mainly of blocks with an alternating sequence of mannuronic and guluronic acid residues (MG blocks) whereas the insoluble fraction consists of homopolymeric blocks of mannuronic acid (MM blocks) and guluronic acid residues (GG blocks) as illustrated in Scheme 1. The fractionation process was achieved through collecting the oligomannuronic (MM) and oligoguluronic (GG) homopolymeric blocks that remained intact as residues via centrifugation. This separated mixture of homopolymeric blocks was resuspended in water and then dissolved through neutralization with dilute alkali $(\mathrm{NaOH}, 0.3 \mathrm{M})$. Afterwards, the oligoguluronate blocks were precipitated and collected leaving the oligomannuronate blocks in solution via adjusting the $\mathrm{pH}$ value of the homopolymeric mixtures at 2.85 .

3.2. Synthesis of PEG-Cs Graft Copolymer. The PEG-g-Cs copolymer was synthesized through a modified method reported in our earlier study [25]. The copolymer synthesis started with the modification of $\mathrm{m}-\mathrm{PEG}$ into carboxylcapped $\mathrm{m}$-PEG precursor (m-PEG-COOH) using succinic anhydride. The prepared $\mathrm{m}-\mathrm{PEG}-\mathrm{COOH}$ was characterized using EA and FTIR. The free $\mathrm{NH}_{2}$ groups of $\mathrm{Cs}$ were protected through phthaloylation process using phthalic anhydride to produce N-phthaloyl Cs (NPHCs). The preparation of NPHCs was confirmed by FTIR through appearance of peaks at 1395 and $732 \mathrm{~cm}^{-1}$ which stand for the "aromatic $\mathrm{C}=\mathrm{C}$ " and "aromatic $\mathrm{C}-\mathrm{H}$ " bonds of the phthaloyl moieties, respectively. Afterwards, grafting of mPEG-COOH onto NPHCs was carried out in DMF, and the grafting \% of the resulting PEG-g-NPHCs was found to be about $9.3 \%$. The resulting PEG-g-NPHCs copolymer was also characterized by EA and FTIR. The PEG-g-Cs copolymer was then produced through the dephthaloylation of the protected $\mathrm{NH}_{2}$ groups of PEG-g-NPHCs copolymer using hydrazine monohydrate. The overall synthesis of the PEG-g-Cs copolymer is demonstrated in Scheme 2. The synthesis of the PEG-g-Cs copolymer was confirmed by comparing the FTIR spectra of the copolymer with those of the starting material (Cs) as shown in Figure 1. The synthesis of the PEG-g-Cs was also proved by studying its thermal behavior in comparison with that of starting materials, PEG-COOH and Cs, as apparent in Figure 2. For instance, the DSC thermogram of PEG-COOH (Figure 2(a)) showed an endothermic band at about $65^{\circ} \mathrm{C}$ which stands for its melting process. In case of the Cs thermogram (Figure 2(b)), it shows an endothermic peak started at about 

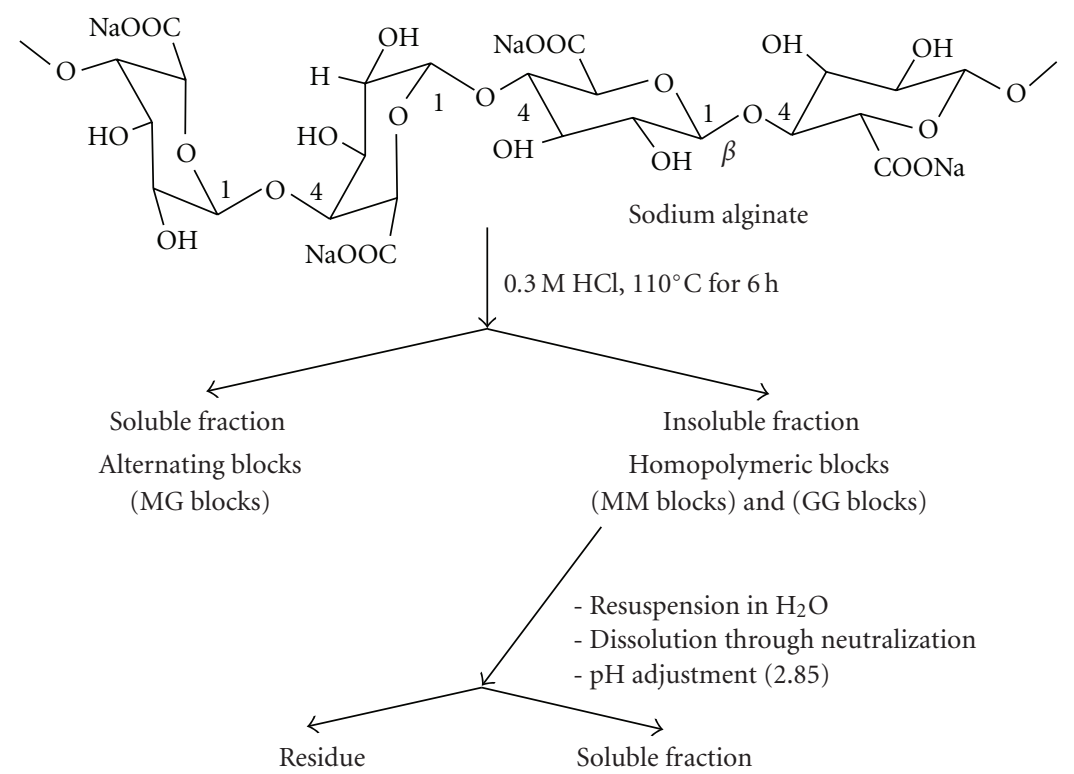

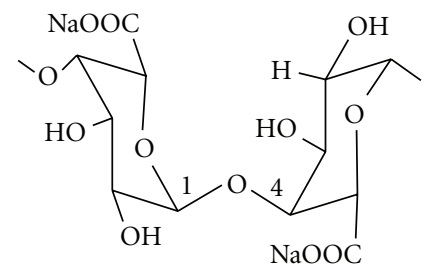

Oligoguluronate (G-blocks)

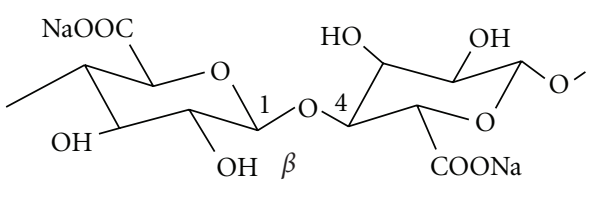

Oligomannuronate (M-blocks)

Scheme 1: Schematic illustration of the preparation of oligoguluronate blocks (G-blocks).

$90^{\circ} \mathrm{C}$, which can be ascribed to the loss of bound water. The Cs thermogram shows also an exotherm at $312^{\circ} \mathrm{C}$ which may be attributed to the decomposition of the glucosamine units $[26,27]$. The thermogram of PEG-g-Cs (Figure 2(c)) shows an endothermic peak at $55^{\circ} \mathrm{C}$ which can be attributed to the melting of the grafted PEG side chains. Also, an endotherm was observed at $119^{\circ} \mathrm{C}$ which is due to the loss of bound water. The exotherms appearing at 261 and $310^{\circ} \mathrm{C}$ may be ascribed to the crystallization and decomposition of the PEG-g-Cs copolymer, respectively.

\subsection{Aerosol-Assisted Preparation of the Magnetic Hydrogel} Nanoparticles. The magnetic hydrogel nanoparticles developed in this study were obtained using a novel spray method as illustrated in Figure 3(a). The system consists of a regulated source of compressed air connected directly to an air-jet nebulizer that delivers the polymeric solutions as sprayed microdroplets to a feed zone comprising tubing carrying droplets. This tubing was assembled to one or more of aerodynamic aerosol classifiers connected to a secondary vacuum system responsible of transporting the sprayed droplets to the crosslinker solution (collecting fluid). Then, an alternate empty glass collector was directly assembled to the primary vacuum system to recover the large hydrogel nanoparticles dragged from the secondary vacuum system.

The prepared hydrogel nanoparticles are based on either sodium alginate or a combination of sodium alginate with oligoguluronate residue (G-blocks) aerosolized as microdroplets followed by either ionotropic gelation in aqueous $\mathrm{Ca}^{2+}$ solution or both ionotropic and polyelectrolyte complexation in $(1: 1)$ aqueous solution of PEG-g-Cs/ $\mathrm{CaCl}_{2}$ as crosslinkers as illustrated in Figure 3(b). In case of the ionotropic gelation of sodium alginate with $\mathrm{Ca}^{2+}$, the carboxylate groups of subsequent guluronate $(G)$ units in the alginate chains have suitable geometry and spacing for the binding with the $\mathrm{Ca}^{2+}$ ions [28]. This affinity of the $\mathrm{G}$ units towards binding with the $\mathrm{Ca}^{2+}$ has been found to be higher than their mannuronate (M) counterparts [29]. Also, this type of binding has been described by the "egg-box" model $[15,16]$. Therefore, the gel strength of alginate-based hydrogels depends mainly on the $\mathrm{G}$ content of the alginate in addition to the other factors such as the calcium ion concentration and the molecular weight and concentration of the alginate. In a recent study [14], free G-blocks were added to alginate solutions and the effects of this addition onto the gel strength, gelling kinetics, and syneresis of the formed alginate-based hydrogels were investigated. The results of the study showed that the free G-blocks are involved in the $\mathrm{Ca}^{2+}$ mediated bonds formed between guluronic acid sequences within the polymeric alginates (Figure 3(b)) [14]. In other words, the added oligoguluronate (G-blocks) competes with the alginate chains for the $\mathrm{Ca}^{2+}$ ions. However, the study indicated that the presence of free G-blocks led to an increase in the gelation time. Also, the gel strength of the formed 


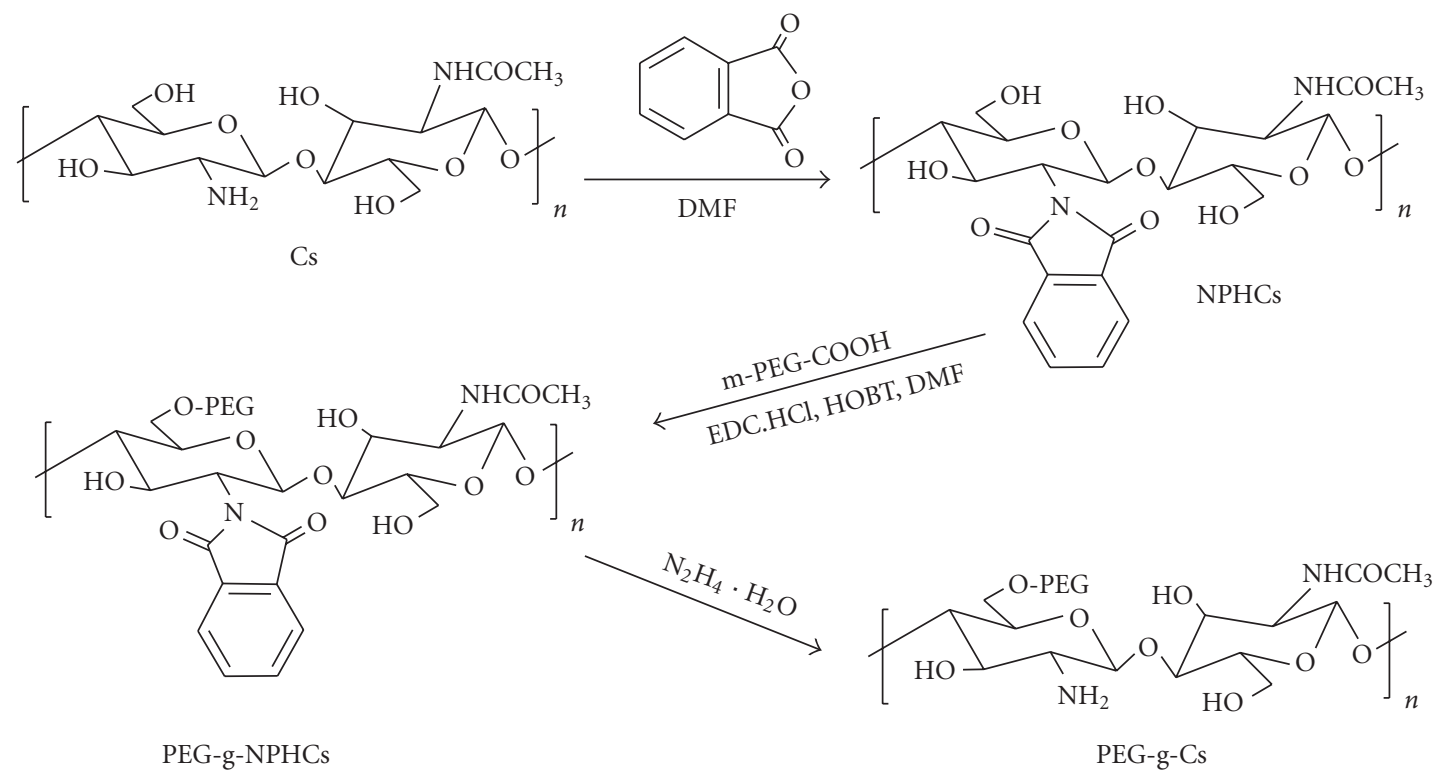

Scheme 2: Schematic illustration of the synthesis of PEG-g-Cs copolymer.

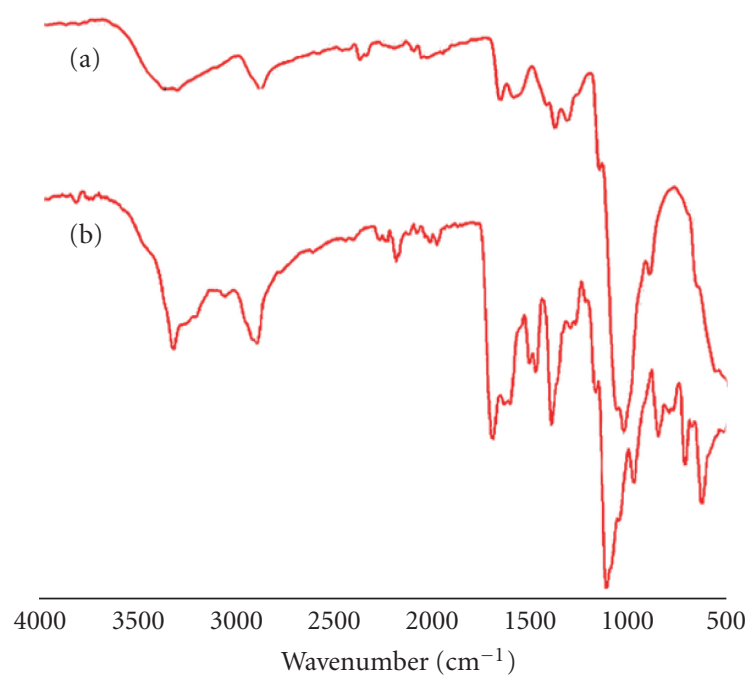

FIGURE 1: FTIR spectra of (a) Cs as compared to (b) the synthesized PEG-g-Cs copolymer.

alginate hydrogels was found to decrease with increasing amount of the free G-blocks. This reduction in the gel strength can be attributed to the lower molecular weight and viscosity of the added oligoguluronate (G-blocks) as compared to the polymeric alginate.

The developed hydrogel nanoparticles showed a considerable magnetically responsive nature as apparent in Figure 4 . The figure reveals the relatively fast response of the hydrogel nanoparticles in their aqueous suspension to an external magnetic field.

3.4. Particle Size Analysis of Hydrogel Nanoparticles. Development of magnetic nanoparticles with appropriate size

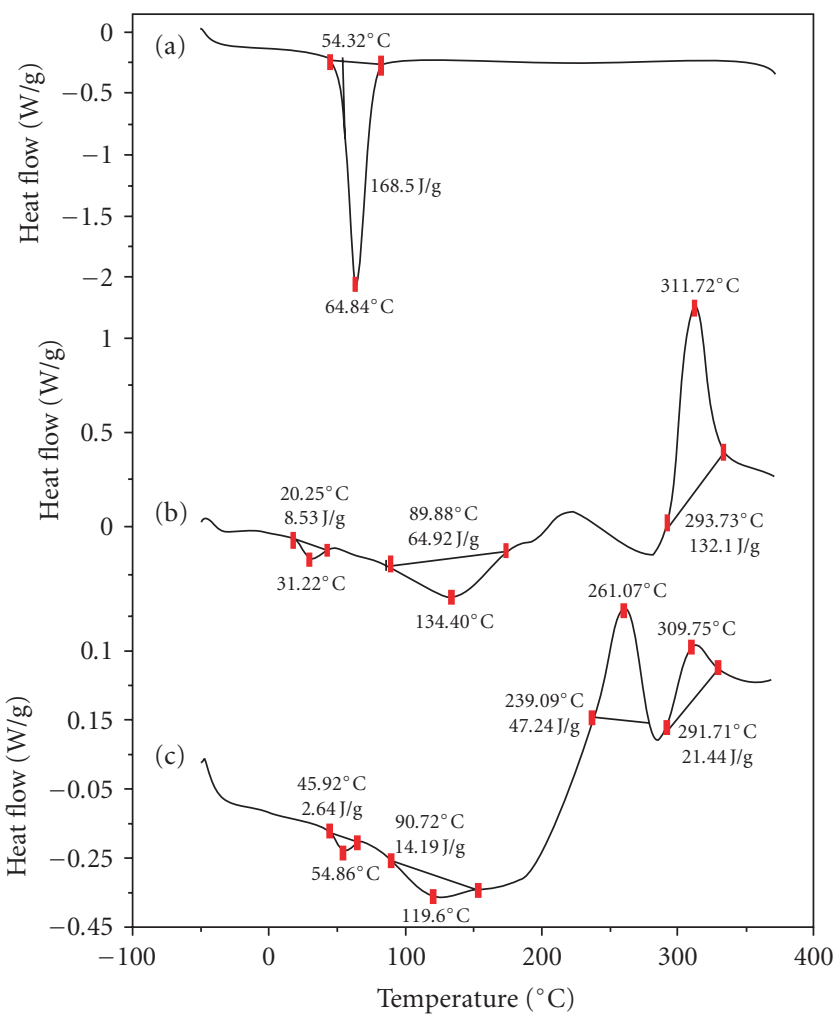

FIgure 2: DSC characterizations of the synthesized PEG-g-Cs copolymer (c) as compared to the starting materials; (a) PEG$\mathrm{COOH}$ and (b) Cs.

represents an important issue in many applications. As shown in Table 1, the size of the developed magnetic hydrogel nanoparticles was found to be in the range of $811 \pm 162$ to $941 \pm 2 \mathrm{~nm}$ as determined by dynamic light scattering 


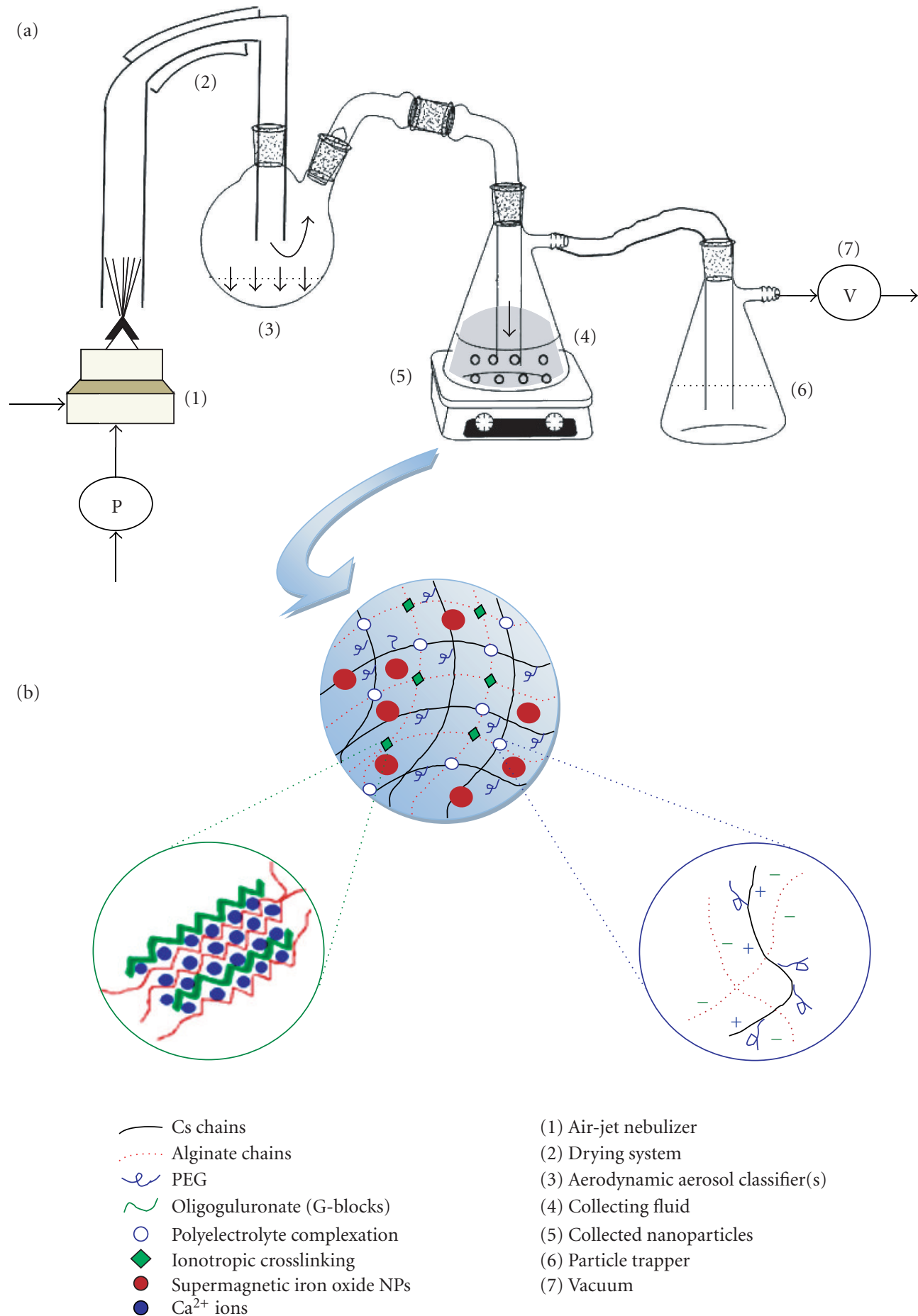

FIGURE 3: (a) An illustration of the new spray gelation-based method used in the development of the hydrogel nanoparticles. (b) Schematic illustration of the developed magnetically responsive hydrogel nanoparticles.

(DLS). From the table, it seems, in most formulations, that crosslinking of the hydrogel nanoparticles with a mixture of $\mathrm{Ca}^{2+} /$ PEG-g-Cs $(1: 1)$ relatively reduces the particle size as compared to the particles crosslinked with only one crosslinking agent $\left(\mathrm{Ca}^{2+}\right)$. For instance, the particles IIIA (crosslinked with $\mathrm{Ca}^{2+}$ only) showed an average size of $941 \pm 2 \mathrm{~nm}$ when analyzed by DLS. This size was reduced to $886 \pm 2 \mathrm{~nm}$ in case of the particles IIIB (crosslinked with $\mathrm{Ca}^{2+} / \mathrm{PEG}$-g-Cs). This effect of the crosslinking agents on the size of the developed hydrogel nanoparticles was found to be statistically significant $(P=.0415)$. Also, it was noted from the size data that, in general, increasing the percentage of alginate in the magnetic hydrogel nanoparticles has no significant effect on the particle size $(P=.7702)$. This can 


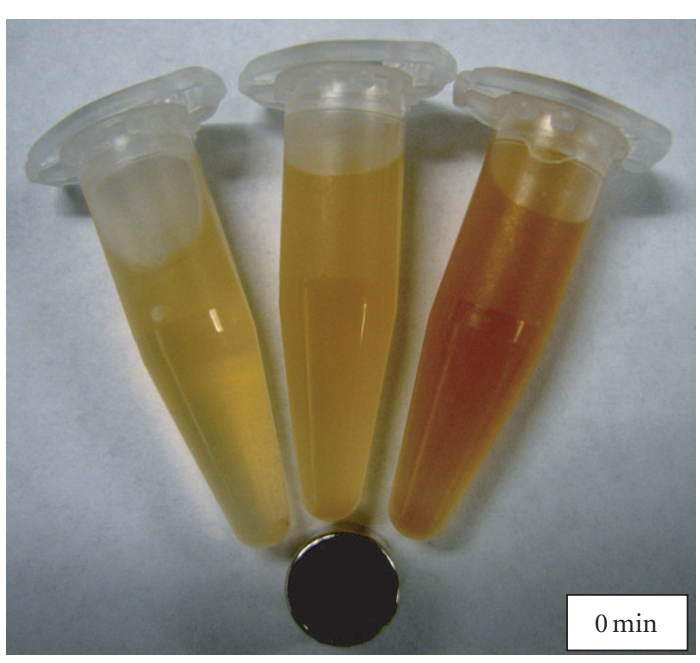

(a)

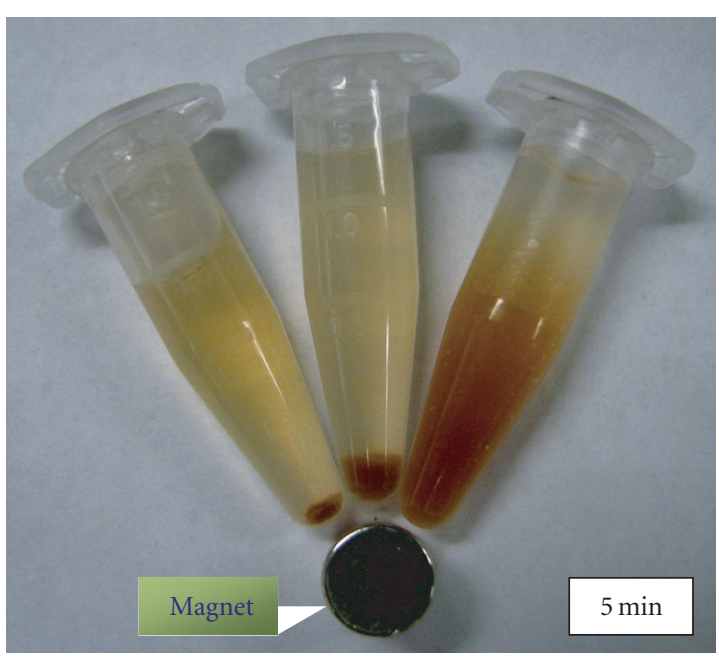

(b)

FIGURE 4: Photographs showing the magnetic nature of the developed hydrogel nanoparticles (aqueous suspensions of different concentrations).

be attributed to the fact that not only sodium alginate but also the oligoguluronate (G-blocks) is participating in the crosslinking process either ionotropically with the $\mathrm{Ca}^{2+}$ ions or through polyelectrolyte complexation with the cationic PEG-g-Cs copolymer [14]. These effects of both the type of crosslinking agent and alginate \% on the particle size were also confirmed through the particle size determinations using light microscopy as shown in Table 1. Further studies have been initiated to assess particle size modulation using different experimental parameters (as discussed below).

\subsection{Scanning Electron Microscopy Analysis of the Hydrogel} Nanoparticles. Figure 5 shows the scanning electron micrographs of some of the developed magnetic hydrogel nanoparticles. From the figure, the hydrogel nanoparticles have, in general, spherical shapes with relatively smooth surfaces. However, the extent of surface regularity and smoothness seems to vary upon changing the type of the used crosslinking agent. For instance, the hydrogel nanoparticles developed using only $\mathrm{Ca}^{2+}$ as a crosslinking agent (Figure 5(a)) showed a spherical morphology with highly smooth, dense, and integrated surfaces. However, nanoparticles showed a relatively rough surface when crosslinked with a mixture of $\mathrm{Ca}^{2+}$ and PEG-g-Cs (Figure 5(b)). This surface morphology of the nanoparticles crosslinked with both $\mathrm{Ca}^{2+}$ and PEG-gCs can be seen more clearly in Figure 5(c).

3.6. Transmission Electron Microscopy Analysis of the Hydrogel Nanoparticles. Figure 6(a) shows the TEM images of the developed magnetic hydrogel nanoparticles. The figure reveals a relatively homogenous distribution of the magnetic cores (SPIONs) throughout the matrix of the hydrogel particles. The content of the iron cores (atomic \%) within the hydrogel nanoparticles was also estimated with the aid of energy-dispersive X-ray spectroscopy (EDS) as illustrated in Figure 6(b). From the figure, the atomic iron content (\%) was found to be about $20 \%$.

\subsection{Dynamic Swelling Study of the Hydrogel Nanoparticles.} The swelling patterns of the prepared magnetic hydrogel nanoparticles in PBS, pH 7.4 are illustrated in Figures 7 and 8 . The swelling data was obtained by measuring the increase in the diameters $(d, \mathrm{~nm})$ of the hydrogel nanoparticles with time using light microscopy. From the swelling data, it seems that the hydrogel nanoparticles prepared using a mixture of $\mathrm{Ca}^{2+}$ and PEG-g-Cs as a crosslinking agent attained relatively lower swelling values than the corresponding formulations that crosslinked only with $\mathrm{Ca}^{2+}$. For instance, after $10 \mathrm{~min}$ of swelling, the hydrogel nanoparticles, IIIB (crosslinked with both $\mathrm{Ca}^{2+}$ and PEG-g-Cs), attained a swelled size of $1592 \pm$ $126 \mathrm{~nm}$ as compared to the nanoparticles, IIIA (crosslinked with $\mathrm{Ca}^{2+}$ ), which attained a swelled size of $1639 \pm 75 \mathrm{~nm}$. This behavior can be attributed to increasing the crosslinking extent of the particles upon using two types of crosslinkings (ionotropic and polyelectrolyte complexation) than using only ionotropic crosslinking with $\mathrm{Ca}^{2+}$. This effect of the crosslinker type on the swelling values attained at equilibrium was found to be statistically significant $(P=.0001)$. Moreover, from the swelling patterns shown in both Figures 7 and 8, although both alginate and oligoguluronate (Gblocks) contribute in the crosslinking process, it seems, in general, that increasing the alginate percentage in the developed nanoparticles tends to increase the crosslinking extent and consequently reduces the swelled sizes. This confirms the higher ability of the alginate as compared to the G-blocks to interact with the crosslinking agents, which is in agreement with the literature [14]. Figure 9 shows some microscopic images that illustrate the differences in size of some developed magnetic hydrogel nanoparticles, including before and after attaining the equilibrium swelling. 


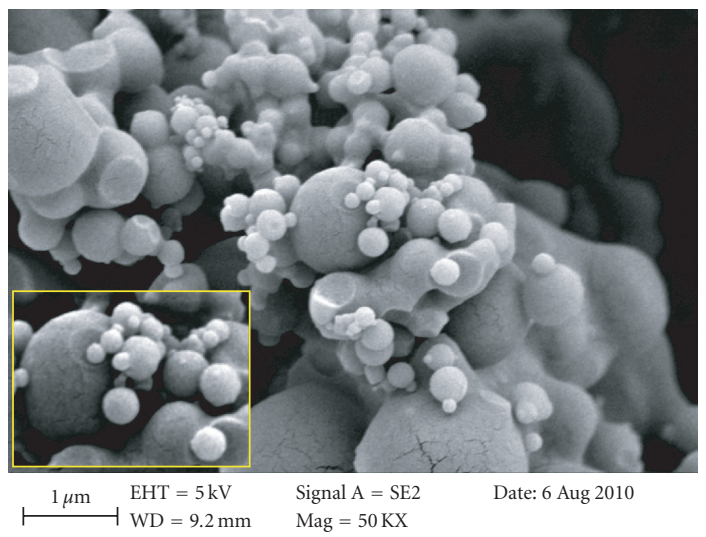

(a)

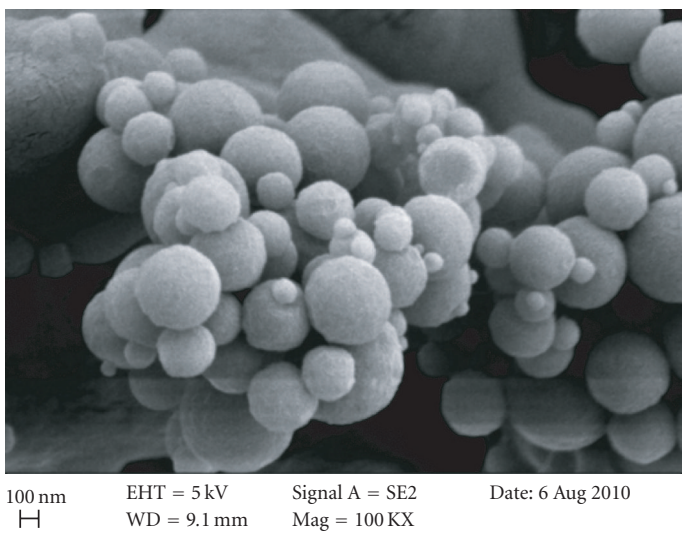

(b)

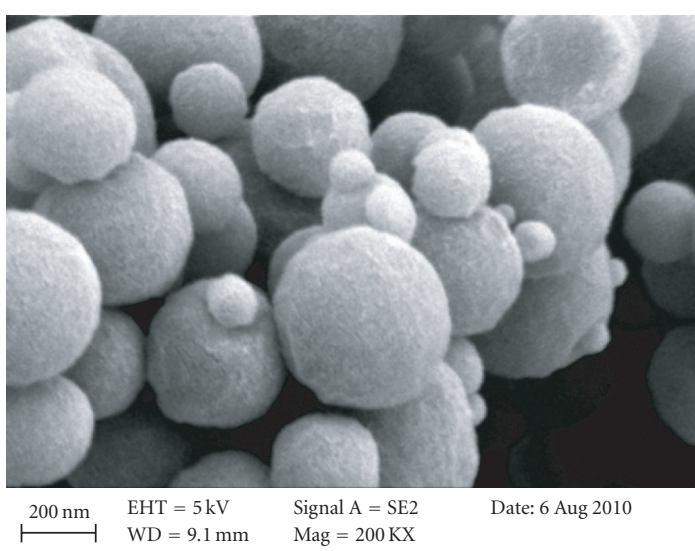

(c)

FIGURE 5: Scanning electron micrographs of some developed magnetic hydrogel nanoparticles; IIIA (a) and IIIB (b and c) showing the spherical nature of the developed nanoparticles.

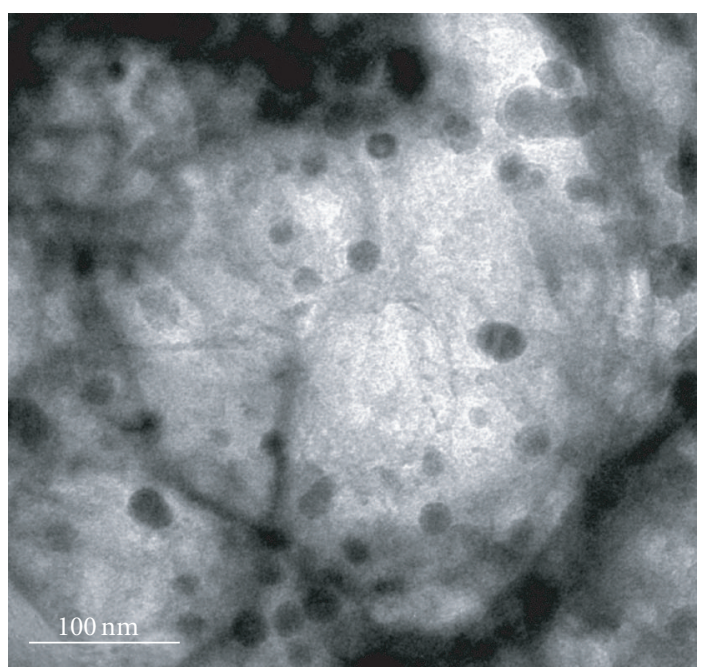

(a)

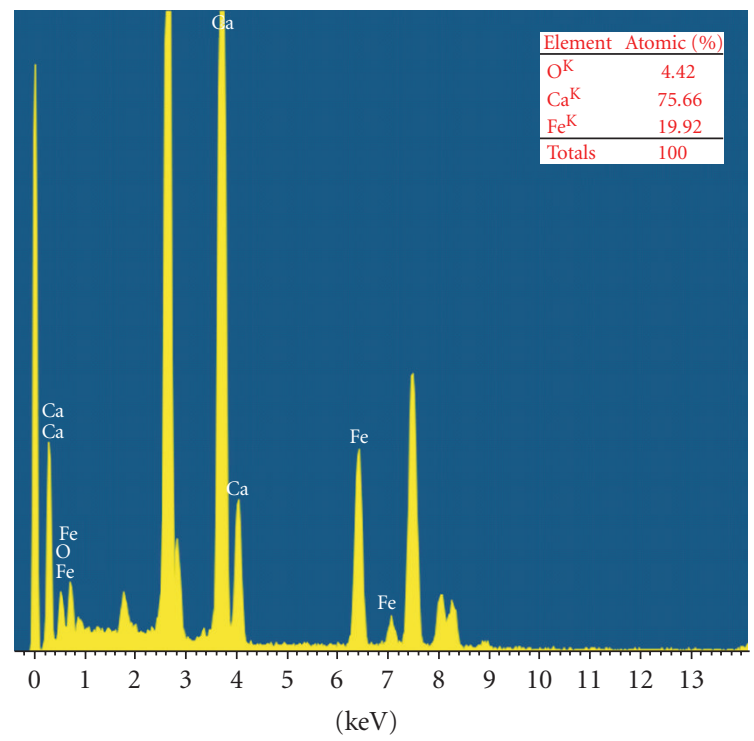

(b)

Figure 6: (a) Transmission electron micrographs (TEM) of the developed magnetic hydrogel nanoparticles. (b) The iron and calcium contents of the magnetic hydrogels as determined by the EDS. 


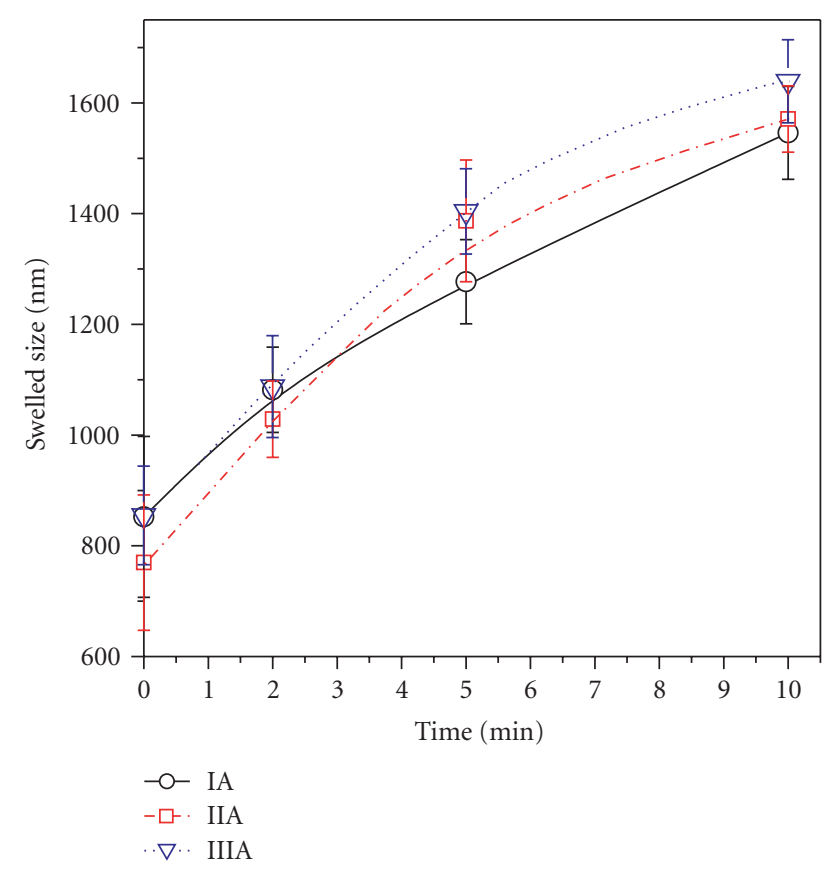

FIGURE 7: Dynamic swelling of the magnetic hydrogel nanoparticles crosslinked with $\mathrm{CaCl}_{2}$ in PBS, 7.4.

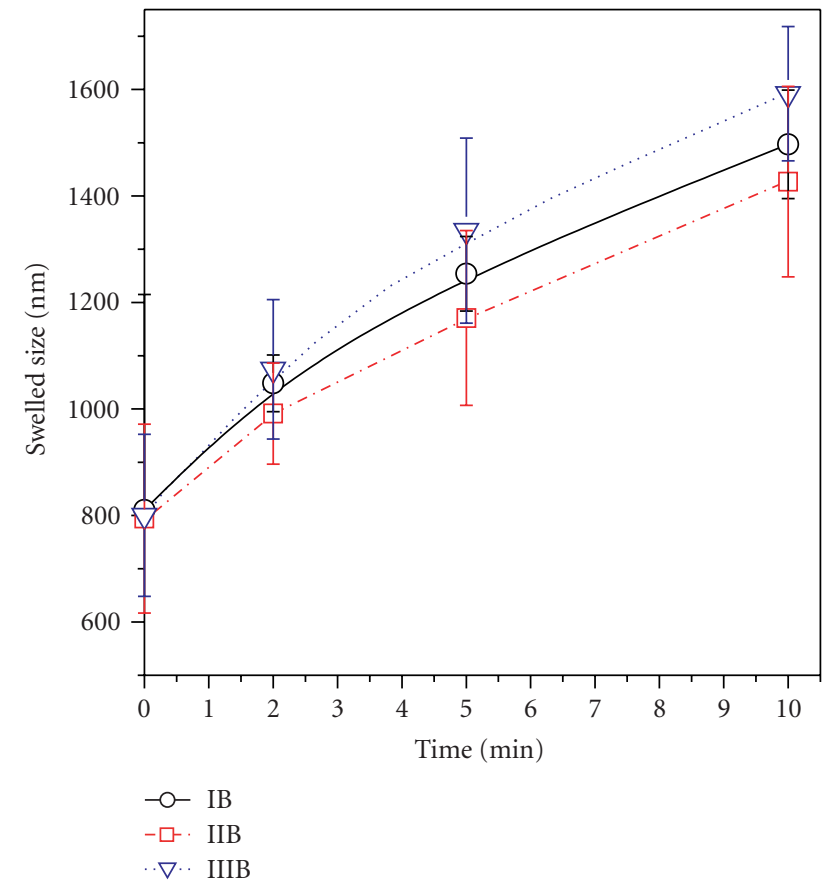

FIGURE 8: Dynamic swelling of the magnetic hydrogel nanoparticles crosslinked with $\mathrm{CaCl}_{2} / \mathrm{PEG}$-g-Cs $(1: 1)$ in PBS, 7.4.

3.8. Analysis of the Characteristics of the Magnetic Hydrogel Nanoparticles by a $2^{k}$ Factorial Design. A discontinuous $2^{k}$ factorial design was performed to investigate the effect of the alginate $\%$ and type of crosslinking agent and their interaction on the characteristics of prepared magnetic hydrogel nanoparticles. The analysis was performed using Pareto

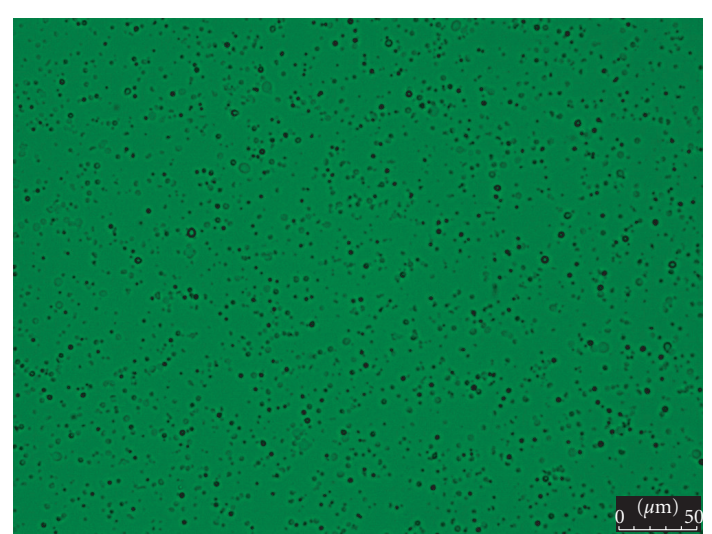

(a)

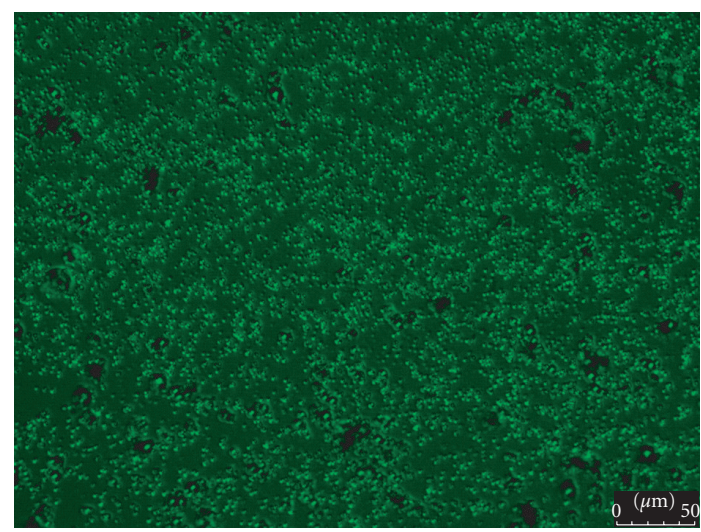

(b)

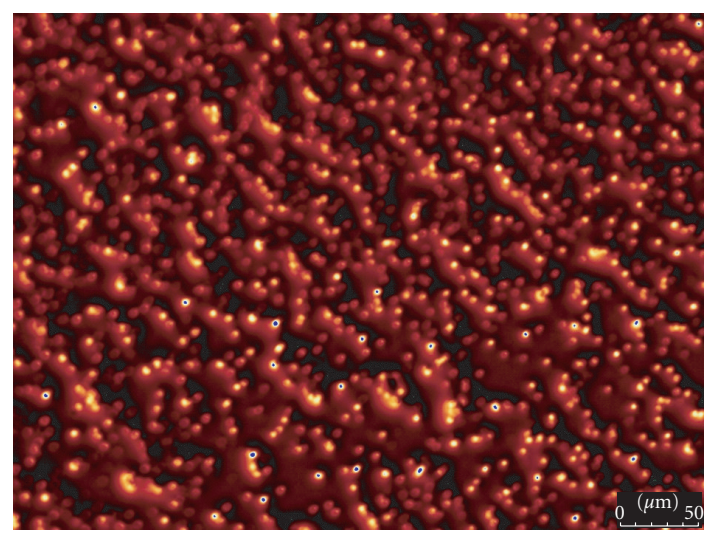

(c)

Figure 9: Microscopic images illustrating the differences in size of some developed magnetic hydrogel nanoparticles (a) dry IIIB NPs, (b) dry IIIA NPs, and (c) swelled IIIA NPs in PBS, pH 7.4.

charts, response surface methodology, and the contour of estimated response surface for three levels of variations in the alginate $(\%)$ factor $(70,80$, and $100 \%$, coded as $-1,0$, and +1 , resp.) plus two levels of variations in the second factor, the crosslinker type $\left(\mathrm{Ca}^{2+}\right.$ and $\mathrm{Ca}^{2+} /$ copolymer, coded as -1 and +1 , resp.). Both the particle size and equilibrium swelling values were used as response variables. The analysis 


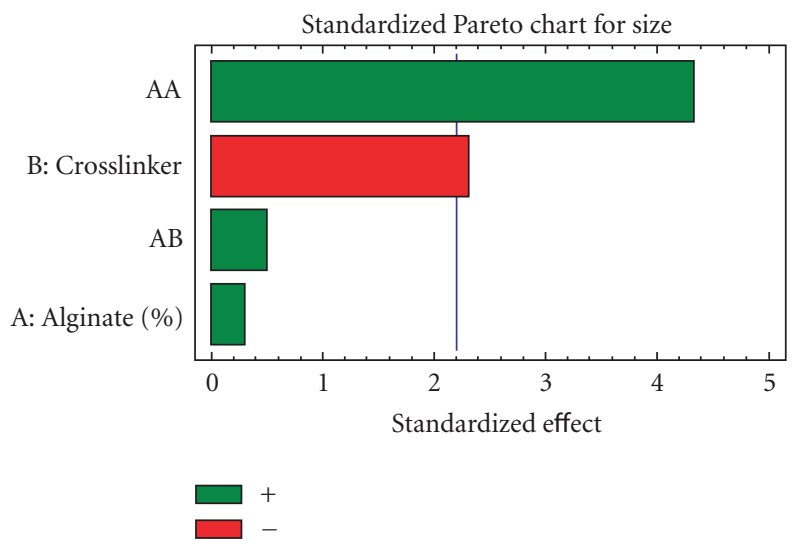

(a)

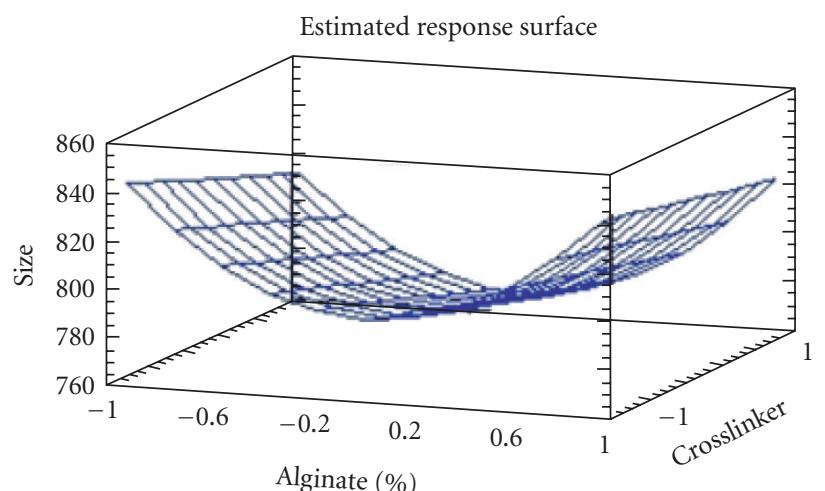

(b)
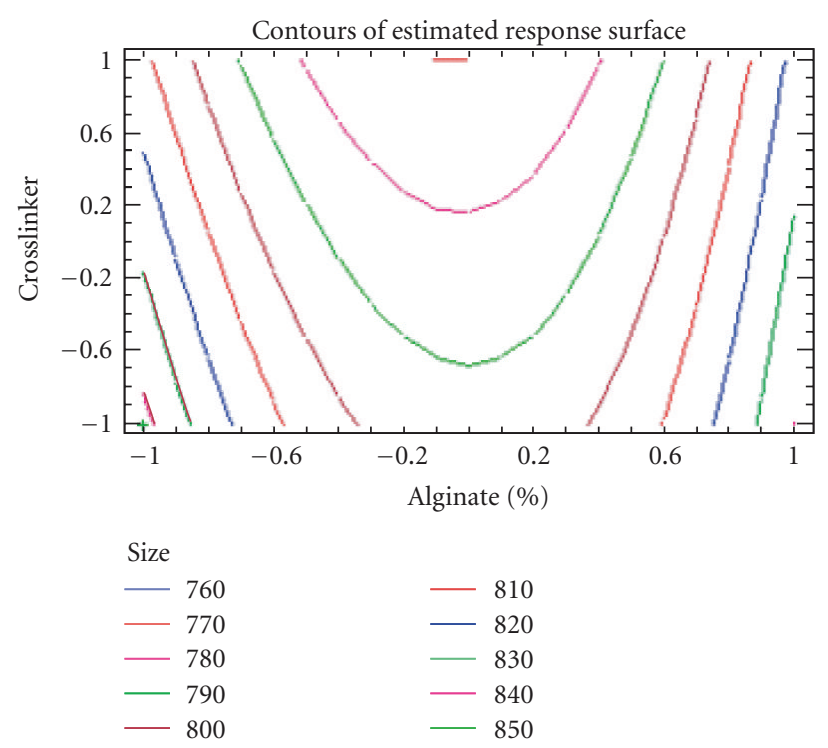

(c)

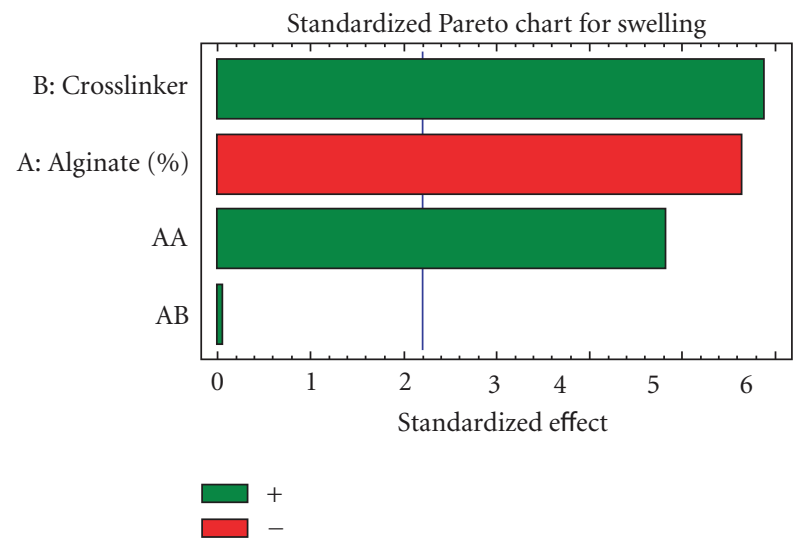

(d)

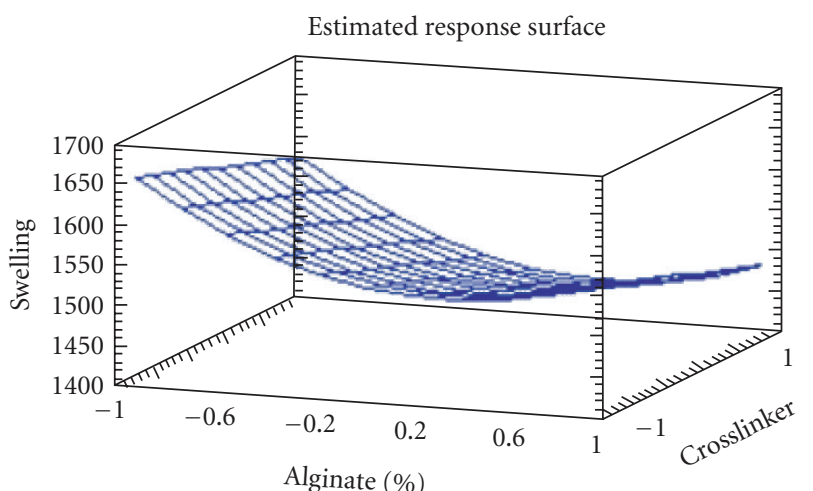

(e)

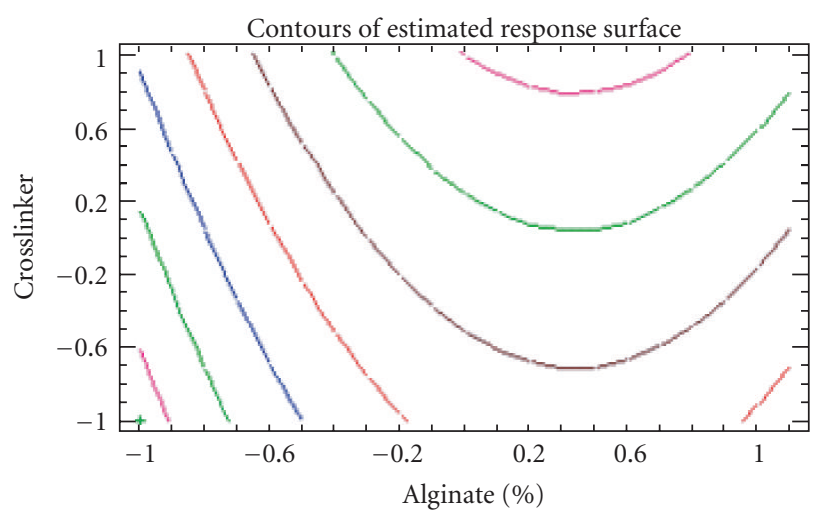

Swelling

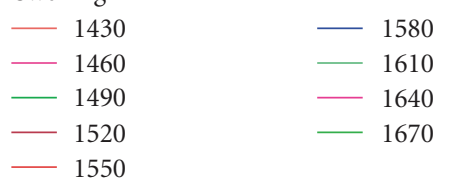

(f)

FigURE 10: Statistical analysis of the effect of the formulation's parameters on both particle size and equilibrium swelling of the developed magnetic hydrogel nanoparticles. 
of variance for both particle size and equilibrium swelling was performed by Statgraphics 5.0, and the most influential factors were estimated at $95 \%$ confidence level.

In case of particle size, as apparent from the standardized Pareto chart (Figure 10(a)), the type of crosslinking agent can be considered the main factor that controls the size of the developed magnetic hydrogel nanoparticles, but as mentioned previously, these differences in particle size do not appear to be practically significant. The surface response methodology was also applied to investigate the way in which the particle size would respond to the parameters variations (Figure 10(b)). The diagram reveals that the smallest particle sizes were obtained when the alginate $\%$ was maintained between -0.2 and +0.2 (knowing that -1 and +1 correspond to $70 \%$ and $100 \%$ alginate, resp.) with the use of a crosslinker mixture (coded as +1$)$. These obtained results are consistent with the particle size data determined experimentally and can be used in future studies to design experimental parameters that may result in a wider range of particle sizes produced. These parameters include the following, among other factors, different atomizer output particle size, drying/conditioning of aerosol prior to crosslinking, concentrations of the hydrogel components, and reaction temperatures.

In the case of the equilibrium swelling, as shown in the standardized Pareto chart (Figure 10(d)), the type of crosslinking agent can be considered the major factor that controls the equilibrium swelling of the hydrogel nanoparticles, followed closely by the alginate \%. Hence, variation of any of these two parameters would likely have a more relevant influence on the swelling values attained at equilibrium. As apparent in the surface response diagram (Figure 10(e)), the lowest swelling values were attained when the alginate \% was maintained in its higher value $(+1$ corresponding to $100 \%$ ) with the use of a $\mathrm{Ca}^{2+}$ /copolymer mixture as a crosslinking agent (coded as +1 ). These results are in agreement with the equilibrium swelling data as determined experimentally.

\section{Conclusion}

A new series of magnetically responsive "smart" hydrogel nanoparticles based on alginate-oligoguluronate was developed using a novel spray gelation-based method. The magnetic nanoparticles showed promising characteristics which can be further optimized by controlling the parameters of the used new method in addition to controlling the relative compositions of the polymeric components.

\section{References}

[1] Y. Qiu and K. Park, "Environment-sensitive hydrogels for drug delivery," Advanced Drug Delivery Reviews, vol. 53, no. 3, pp. 321-339, 2001.

[2] M. A. Morales, P. V. Finotelli, J. A. H. Coaquira et al., "In situ synthesis and magnetic studies of iron oxide nanoparticles in calcium-alginate matrix for biomedical applications," Materials Science and Engineering C, vol. 28, no. 2, pp. 253-257, 2008.
[3] D. K. Kim, Y. Zhang, J. Kehr, T. Klason, B. Bjelke, and M. Muhammed, "Characterization and MRI study of surfactantcoated superparamagnetic nanoparticles administered into the rat brain," Journal of Magnetism and Magnetic Materials, vol. 225, no. 1-2, pp. 256-261, 2001.

[4] C. H. Reynolds, N. Annan, K. Beshah et al., "Gadoliniumloaded nanoparticles: new contrast agents for magnetic resonance imaging," Journal of the American Chemical Society, vol. 122, no. 37, pp. 8940-8945, 2000.

[5] A. S. Lübbe, C. Bergemann, H. Riess et al., "Clinical experiences with magnetic drug targeting: a phase I study with 4 ' epidoxorubicin in 14 patients with advanced solid tumors," Cancer Research, vol. 56, no. 20, pp. 4686-4693, 1996.

[6] C. Bergemann, D. Müller-Schulte, J. Oster, L. À Brassard, and A. S. Lübbe, "Magnetic ion-exchange nano- and microparticles for medical, biochemical and molecular biological applications," Journal of Magnetism and Magnetic Materials, vol. 194, no. 1, pp. 45-52, 1999.

[7] D. C. F. Chan, D. B. Kirpotin, and J. P. A. Bunn, "Synthesis and evaluation of colloidal magnetic iron oxides for the site-specific radiofrequency-induced hyperthermia of cancer," Journal of Magnetism and Magnetic Materials, vol. 122, no. 13, pp. 374-378, 1993.

[8] A. Jordan, R. Scholz, P. Wust et al., "Endocytosis of dextran and silan-coated magnetite nanoparticles and the effect of intracellular hyperthermia on human mammary carcinoma cells in vitro," Journal of Magnetism and Magnetic Materials, vol. 194, no. 1, pp. 185-196, 1999.

[9] A. Dyal, K. Loos, M. Noto et al., "Activity of Candida rugosa lipase immobilized on $\gamma-\mathrm{Fe}_{2} \mathrm{O}_{3}$ magnetic nanoparticles," Journal of the American Chemical Society, vol. 125, no. 7, pp. 16841685, 2003.

[10] R. Weissleder, G. Elizondo, J. Wittenberg, C. A. Rabito, H. H. Bengele, and L. Josephson, "Ultrasmall superparamagnetic iron oxide: characterization of a new class of contrast agents for MR imaging," Radiology, vol. 175, no. 2, pp. 489-493, 1990.

[11] K. Thode, M. Lück, W. Schröder et al., "The influence of the sample preparation on plasma protein adsorption patterns on polysaccharide-stabilized iron oxide particles and N-terminal microsequencing of unknown proteins," Journal of Drug Targeting, vol. 5, no. 1, pp. 35-43, 1997.

[12] T. Y. Liu, S. H. Hu, K. H. Liu, D. M. Liu, and S. Y. Chen, "Preparation and characterization of smart magnetic hydrogels and its use for drug release," Journal of Magnetism and Magnetic Materials, vol. 304, no. 1, pp. e397-e399, 2006.

[13] S. L. McGill, C. L. Cuylear, N. L. Adolphi, M. Osiński, and H. D. C. Smyth, "Magnetically responsive nanoparticles for drug delivery applications using low magnetic field strengths," IEEE Transactions on Nanobioscience, vol. 8, no. 1, Article ID 4801978, pp. 33-42, 2009.

[14] T. E. Jørgensen, M. Sletmoen, K. I. Draget, and B. T. Stokke, "Influence of oligoguluronates on alginate gelation, kinetics, and polymer organization," Biomacromolecules, vol. 8, no. 8, pp. 2388-2397, 2007.

[15] E. R. Morris, D. A. Rees, D. Thom, and J. Boyd, "Chiroptical and stoichiometric evidence of a specific, primary dimerisation process in alginate gelation," Carbohydrate Research, vol. 66, no. 1, pp. 145-154, 1978.

[16] G. T. Grant, E. R. Morris, and D. A. Rees, "Biological interactions between polysaccharides and divalent cations: the egg box model," FEBS Letters, vol. 32, no. 1, pp. 195-198, 1973.

[17] N. V. Majeti and R. Kumar, "A review of chitin and chitosan applications," Reactive and Functional Polymers, vol. 46, no. 1, pp. 1-27, 2000. 
[18] Y. Ohya, R. Cai, H. Nishizawa, K. Hara, and T. Ouchi, "Preparation of PEG-grafted chitosan nanoparticles as peptide drug carriers," S.T.P. Pharma Sciences, vol. 10, no. 1, pp. 77-82, 2000.

[19] P. Opanasopit, T. Ngawhirunpat, T. Rojanarata, C. Choochottiros, and S. Chirachanchai, "N-Phthaloylchitosan-g-mPEG design for all-trans retinoic acid-loaded polymeric micelles," European Journal of Pharmaceutical Sciences, vol. 30, no. 5, pp. 424-431, 2007.

[20] C. Prego, D. Torres, E. Fernandez-Megia, R. Novoa-Carballal, E. Quiñoá, and M. J. Alonso, "Chitosan-PEG nanocapsules as new carriers for oral peptide delivery: effect of chitosan pegylation degree," Journal of Controlled Release, vol. 111, no. 3, pp. 299-308, 2006.

[21] Z. Yao, C. Zhang, Q. Ping, and L. Yu, "A series of novel chitosan derivatives: synthesis, characterization and micellar solubilization of paclitaxel," Carbohydrate Polymers, vol. 68, no. 4, pp. 781-792, 2007.

[22] X. Zhang, H. Zhang, Z. Wu, Z. Wang, H. Niu, and C. Li, "Nasal absorption enhancement of insulin using PEG-grafted chitosan nanoparticles," European Journal of Pharmaceutics and Biopharmaceutics, vol. 68, no. 3, pp. 526-534, 2008.

[23] A. Haug, B. Larsen, and O. Smidsrod, "A study of the constitution of alginic acid by partial acid hydrolysis," Acta Chemica Scandinavica, vol. 20, pp. 183-190, 1966.

[24] A. Haug, B. Larsen, and O. Smidsrød, "Uronic acid sequence in alginate from different sources," Carbohydrate Research, vol. 32, no. 2, pp. 217-225, 1974.

[25] I. M. El-Sherbiny, S. McGill, and H. D. C. Smyth, "Swellable microparticles as carriers for sustained pulmonary drug delivery," Journal of Pharmaceutical Sciences, vol. 99, no. 5, pp. 2343-2356, 2010.

[26] L. S. Guinesi and E. T. G. Cavalheiro, "The use of DSC curves to determine the acetylation degree of chitin/chitosan samples," Thermochimica Acta, vol. 444, no. 2, pp. 128-133, 2006.

[27] F. S. Kittur, K. V. H. Prashanth, K. Udaya Sankar, and R. N. Tharanathan, "Characterization of chitin, chitosan and their carboxymethyl derivatives by differential scanning calorimetry," Carbohydrate Polymers, vol. 49, no. 2, pp. 185193, 2002.

[28] M. L. Dheu-Andries and S. Pérez, "Geometrical features of calcium-carbohydrate interactions," Carbohydrate Research, vol. 124, no. 2, pp. 324-332, 1983.

[29] C. A. Steginsky, J. M. Beale, H. G. Floss, and R. M. Mayer, "Structural determination of alginic acid and the effects of calcium binding as determined by high-field n.m.r," Carbohydrate Research, vol. 225, no. 1, pp. 11-26, 1992. 

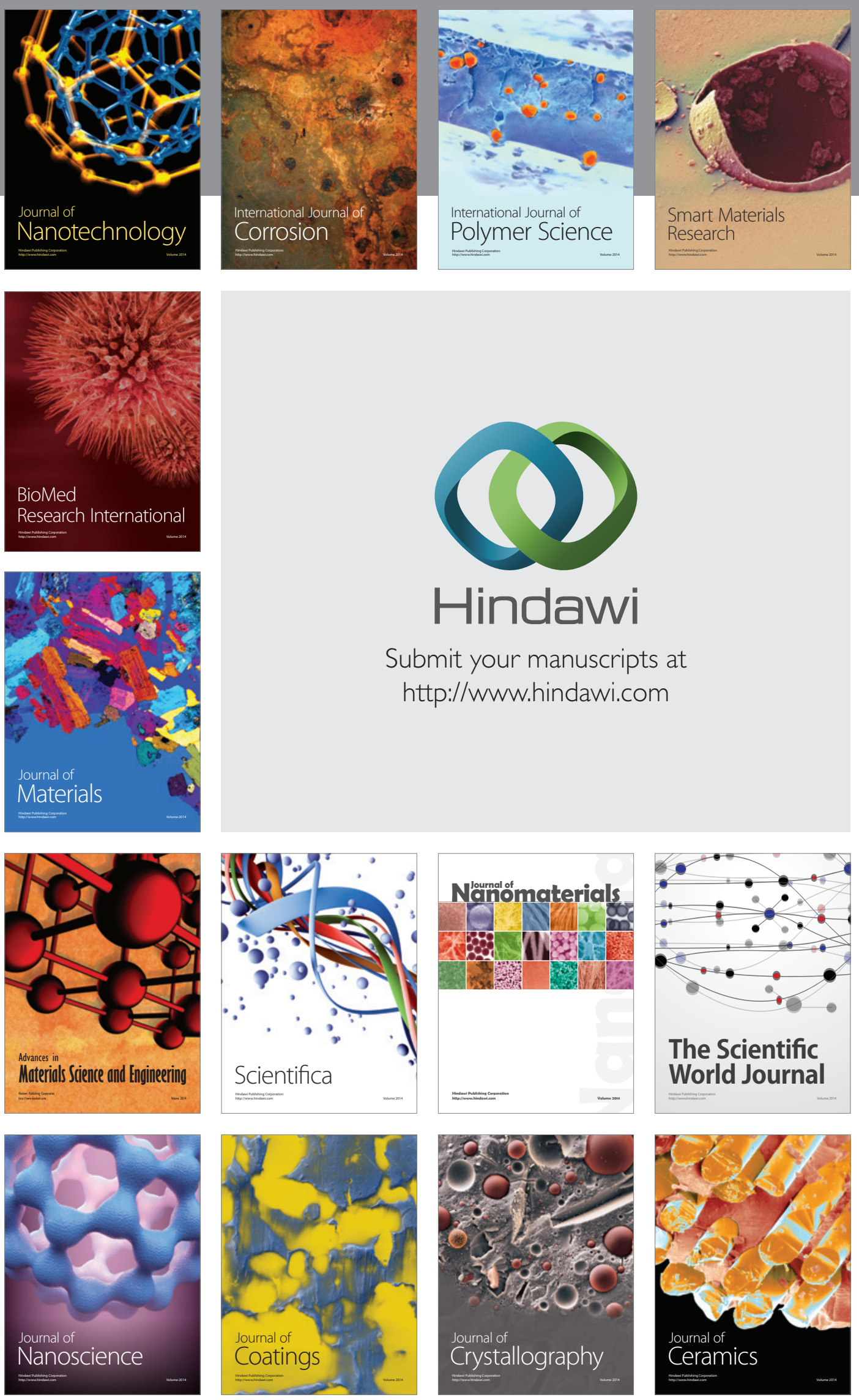

The Scientific World Journal

Submit your manuscripts at

http://www.hindawi.com

\section{World Journal}

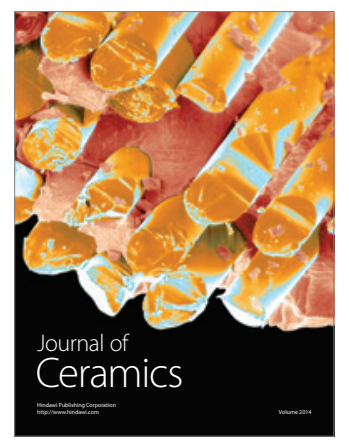

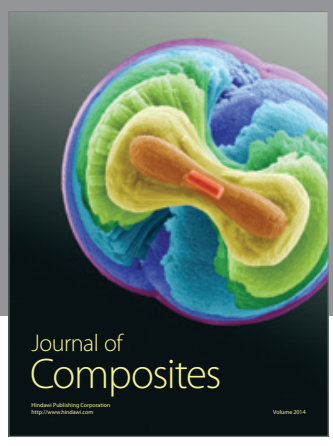
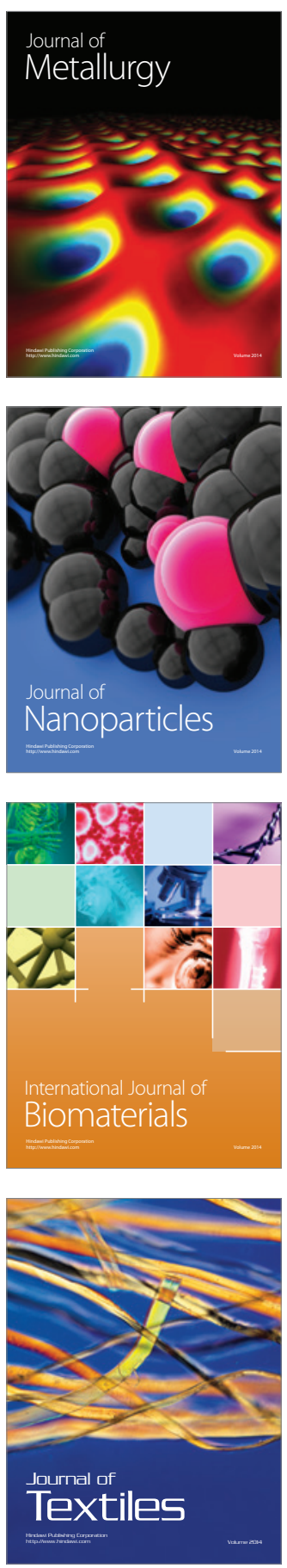